DOI 10.35381/cm.v5i9.154

\title{
Perfil público relacionista del gerente educativo universitario
}

\section{Public relationship profile of the university education manager}

\author{
Luzmila María Valverde Medina \\ luzmilavalverde@yahoo.es \\ Universidad Técnica Luis Vargas Torres de Esmeraldas \\ Ecuador \\ https://orcid.org/0000-0002-8157-4211 \\ Celina Quiñonez Quiñonez \\ celina qq@gmail.com \\ Universidad Técnica Luis Vargas Torres de Esmeraldas \\ Ecuador \\ https://orcid.org/0000-0002-3480-1688 \\ Luis Caicedo Perlaza \\ luiscaicedoperlaza@yahoo.es \\ Universidad Técnica Luis Vargas Torres de Esmeraldas \\ Ecuador \\ https://orcid.org/0000-0001-8696-2697
}

Recibido: 23 de mayo de 2019

Aprobado: 14 de junio de 2019

\section{RESUMEN}

El presente artículo se orientó en caracterizar los enfoques del perfil público relacionista del gerente académico universitario. La metodología utilizó un enfoque descriptivo documental, no experimental, transaccional y de campo. La muestra fue de tipo censal y el instrumento tipo cuestionario. El instrumento fue sometido a la validez de contenido y al cálculo de la confiabilidad con un valor de 0.85 , considerado altamente confiable y cuyo análisis se basó en la estadística descriptiva inferencial. Los resultados indicaron que el $60 \%$ de los gerentes investigados coincidieron en que algunas veces asumen el trabajo en equipo como característica de su perfil relacionista. Entre sus conclusiones se evidencio que se hace imperante una revisión constante de las aptitudes de lederente, empático, creativo, del trabajo en equipo y de los rasgos de responsabilidad entre los gerentes universitarios. 
Descriptores: Perfil público; Relacionista; Gerente universitario; Responsabilidad, Liderente; Empatía; Creatividad; Trabajo en equipo.

\begin{abstract}
This article focused on characterizing the approaches of the public profile related to the university academic manager. To this end, a documentary descriptive, non-experimental, transactional and field methodology was considered. The sample was a censal type. To develop the research, a questionnaire-like instrument and an anecdotal record were applied. The instrument was subjected to the validity of content and the calculation of reliability with a value of 0.85 , considered highly reliable. The analysis was given the inferential descriptive statistic. The results indicated that $60 \%$ of the managers investigated agreed that they sometimes take on teamwork as a characteristic of their relationship profile. Among its conclusions, it is evident that there is a constant review of the skills of heartens, empathetic, creative, teamwork and the traits of responsibility among university managers.
\end{abstract}

Keywords: Public profile; Relationshipist; University manager; Responsibility, Leadership; Empathy; Creativity and teamwork.

\title{
INTRODUCCIÓN
}

El perfil publico relacionista del gerente educativo universitario, ha adquirido en los últimos años, una importancia relevante en el seno de las instituciones universitarias, se hace imperante tener cualidades relacionistas para las correctas relaciones con sus públicos que hacen vida universitaria, se necesitan desarrollar adaptaciones interactivas en beneficio de la gestión gerencial que le corresponde ejecutar. De allí que las teorías de las organizaciones ha ido desarrollando una dinámica de diferentes concepciones que persiguen la mejora de los procesos internos y externos de las organizaciones.

Lo expuesto, demanda de la gerencia universitaria moderna el desarrollo de nuevas capacidades que modelen su perfil gerencial y que estén orientados a la ejecución de planes ajustados a los intereses de la sociedad donde se desenvuelve la institución. Siendo así, se hace necesario fortalecer el perfil público relacionista del gerente específicamente en su proceder como liderente, empático, creativo de su trabajo en 


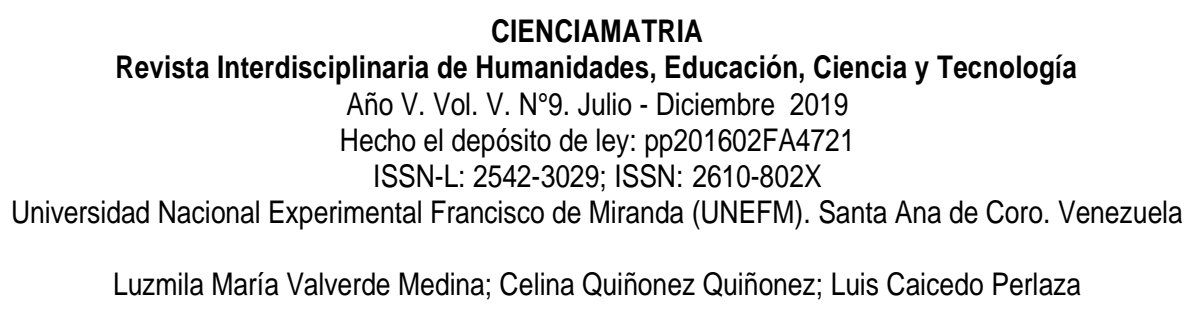

equipo y el nivel de responsabilidad, enfoque necesarios que perfilan y garantizan una gestión gerencial en sintonía a los intereses del entorno universitario.

Por su parte, Miguez (2010), señala que algunos elementos y patrones que se consideran cruciales para un gerente educativo, debe incluir las habilidades de comunicador y persuasión, capacidad de comunicación interpersonal, una naturaleza positiva y proactiva, además de una experiencia laboral variada. Por su parte, Meng y Berger (2010), indican que un excelente gerente abarca un conjunto de habilidades individuales y atributos personales, valores y comportamientos que producen una gerencia consistente. Bajo esta premisa, el estudio se enfocó en caracterizar los enfoques del perfil público relacionista del gerente académico universitario.

\section{DESARROLLO}

Un adecuado perfil profesional, indica Vargas (2009), permite tener una visión integral de un profesional, implica conocer no sólo sus funciones sino también las habilidades, conocimientos, valores y actitudes que deben de regir su labor. Si bien existen elementos que son la base del perfil profesional de todo relacionista público, es innegable que este requiere ser replanteado y actualizado constantemente, de forma que evolucione de la mano de las necesidades de las organizaciones educativas. Para conseguir esto es preciso hacer referencia a las definiciones y teorizaciones que existen, pero estas deben ser complementadas con el conocimiento cotidiano, el que surge del trabajo en las organizaciones y del proceso de aprendizaje de los futuros profesionales. Para este autor, las características de un relacionista público son: creatividad, liderazgo, facilidad para transmitir ideas, capacidad de plasmar ideas por escrito, trabajo en equipo, iniciativa, actitud positiva, disposición a pedir ayuda, tolerancia, visión a futuro y flexibilidad, entre otros.

No obstante, Barquero (2008), plantea que la formación en Relaciones Públicas es una necesidad que se origina en las organizaciones públicas y privadas, y la sociedad actual demanda hoy más profesionales en esta área que en otra época, pues se vive en la 


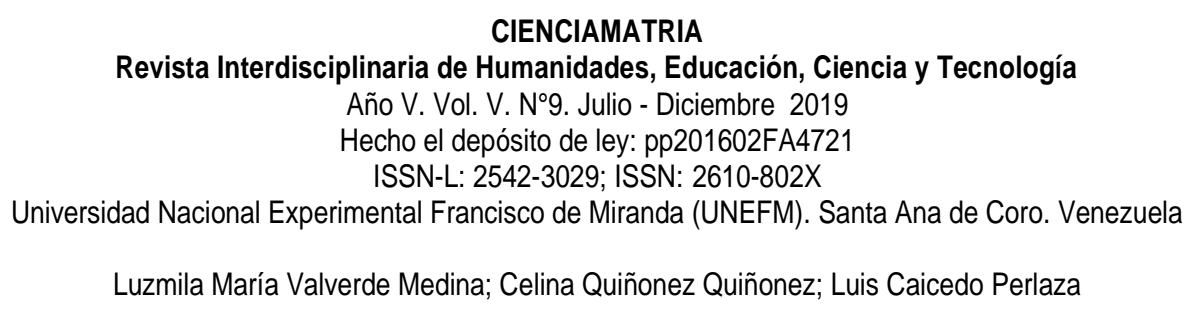

sociedad del conocimiento y de la comunicación. Además, estudia el entorno, analiza la opinión pública e influye en la formación de opinión. Participa e influye en la configuración de la personalidad, identidad, cultura organizacional y comunicación estratégica. Formula la planificación estratégica comunicacional basado en el plan estratégico de la institucion, incluyendo las situaciones conflictivas.

También construye y controla la imagen corporativa garantizando la veracidad y pulcritud de la actuación institucional. Coordina su trabajo permanente con publicidad, planificación y recursos humanos. Participa en la formulación de políticas orientadas a la responsabilidad social y moral de la organización. El mismo autor señala que el perfil del Relacionista Público en el desempeño de sus funciones, será fácil custodio de los intereses de la organización a la cual sirve, ya en forma directa o indirecta, garantizando en su labor una celosa reserva en toda la información a la que tiene acceso y un cuidadoso criterio en la disposición de los elementos que pueda aportar ese conocimiento.

Por su parte, el autor Otero (2009), detalla que el relacionista público debe conocer a la perfección las técnicas más avanzadas de comunicación verbal, ya que se puede valer de ellas como instrumentos imprescindibles para conseguir sus objetivos relacionales. Las tareas que un relacionista público debe manejar para ser un líder eficaz, son: emprender, gestionar, asignar recursos y negociar.

Aunado a lo anterior, Vargas (2009) agrega que, dentro de las características de las Relaciones Públicas, es preciso hacer hincapié en la creatividad como una cualidad pocas veces mencionada, pero de gran importancia dentro de la profesión, los profesionales en particular señalan su preocupación, ya que los estudiantes suelen asumir que dentro del área de la comunicación, las Relaciones Públicas son la única que no requiere de creatividad y que debe convertirse en parte esencial de la profesión y si bien no es una cualidad que se pueda brindar como un conocimiento, debe ser estimulada de manera que perciban la innovación como parte importante de su labor. 


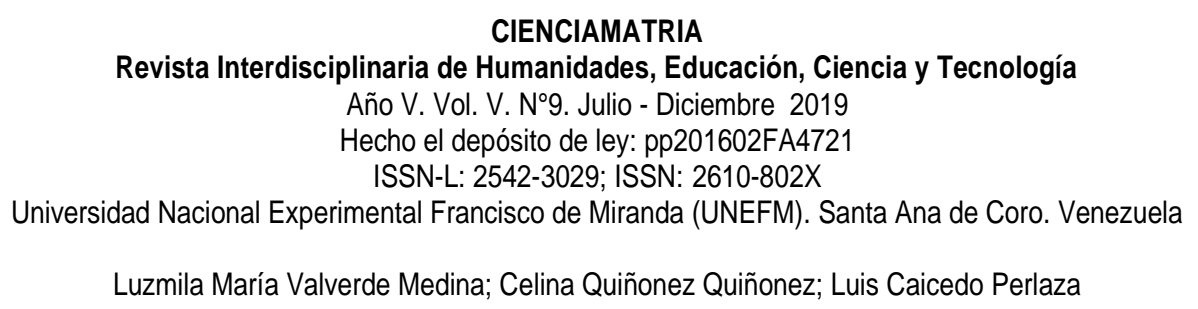

En resumen, un perfil publico relacionista del gerente educativo universitario cuenta con una particularidad, ya que los conocimientos teóricos y técnicos no son suficientes para la formación y desempeño del profesional, es necesario el desarrollo de ciertas cualidades personales que complementan y mejoran la labor del gerente al brindarle más herramientas para cumplir de forma efectiva, creativa y coherente sus objetivos. El mayor reto para este perfil relacionista consiste en consolidar a nivel institucional la importancia de la labor gerencial docente como una inversión que puede ahorrar muchas crisis, desorganización y pérdida de recursos.

Así mismo, Moraima Romero \& Aldana Zavala (2019), destacan que el gerente educativo debe poseer un perfil que abarque lo comunitario, lo cual permite promover estrategias de integración de la institución con la comunidad, promoviéndose una educación de calidad basada en las múltiples interrelaciones sociales. En complemento, Gómez\& Salas de González (2018), destacan que el gerente educativo debe poseer competencias emocionales que le permitan fomentar un clima organizacional asertivo para el establecimiento del compromiso al trabajo por parte de los integrantes de la institución.

Por todo ello, es importante que el gerente fortalezca su perfil publico relacionista a través del liderazgo, empatía, creatividad, trabajo en equipo y responsabilidad porque son las personas encargadas de promover el prestigio o buena imagen de los espacios académicos universitarios mediante el trato personal y la conexión emocional entre instituciones involucradas, docentes, estudiantes y más partes integradas por grupos de seres humanos. Autores como Alles (2013), explican que el perfil publico relacionista del gerente educativo, estar caracterizado por su capacidad de liderente, empatía, creatividad, trabajo en equipo y responsabilidad.

Liderente: según Meléndez (2000), refiere a la persona que ejecuta actividades diarias en una empresa, que son típicas del líder y del gerente, y quien muestra un perfil de actuación que le permite abrirse como exitosamente hacia el futuro, mientras dirige y administra los medios de la organización. Por otro lado, el liderente en sus funciones, 
considera tres escenarios: pasado, presente y futuro. Las experiencias pasadas, ayudan a configurar el presente y a construir las propuestas para el futuro, sin embargo, aferrarse a la idea de repetir tradiciones teóricas o empíricas exitosas del pasado lejano podría no ajustarse a los requerimientos actuales de productividad y competitividad, que constituyen evidencias de acciones que fueron loables en un momento histórico dado. Por otra parte, las actuaciones del pasado reciente, tientan al ejecutor de hoy a repetir sus acciones ante la creencia de logros con baja probabilidad de riesgos y fracasos.

El futuro lejano, encierra el peligro de suponer tanto la relativa inamovilidad del entorno, de las condiciones particulares de la institucion como de la aplicación objetiva de métodos prospectivos que garanticen el éxito a través de la instrumentación de estrategias y tácticas suficientemente razonadas. El futuro cercano, con su sensación de inmediatez, aporta escenarios para soluciones a corto plazo y triunfos que conducen hacia el futuro lejano, siempre que se hayan tomado las previsiones. Cabe destacar, que el liderante, actúa con base en las referencias pasadas, mira hacia el futuro y confronta el presente con decisión. Tal como se observa en el gráfico 1:

\section{Grafico 1. Actuación diaria del Liderente.}

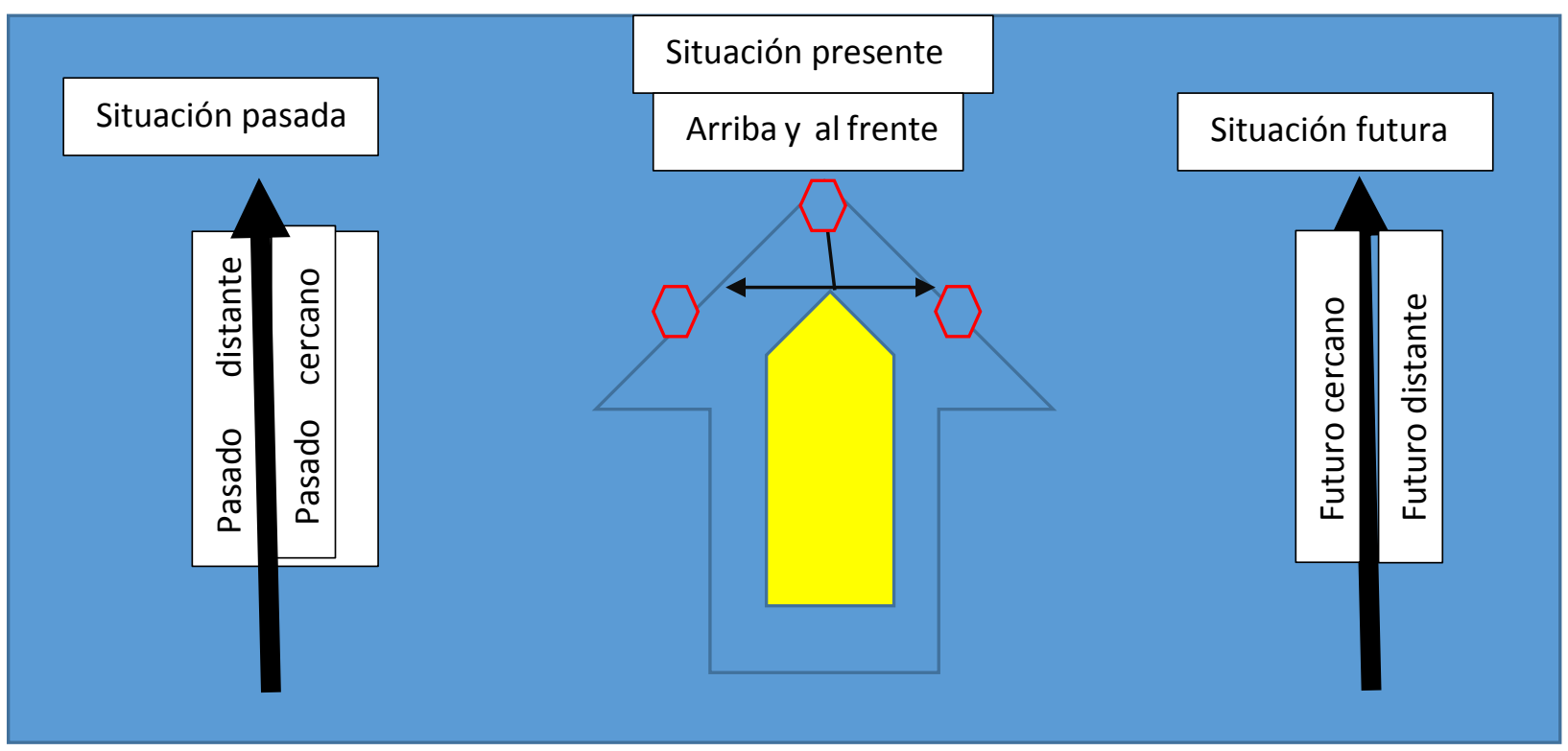

Fuente: Meléndez (2000). 


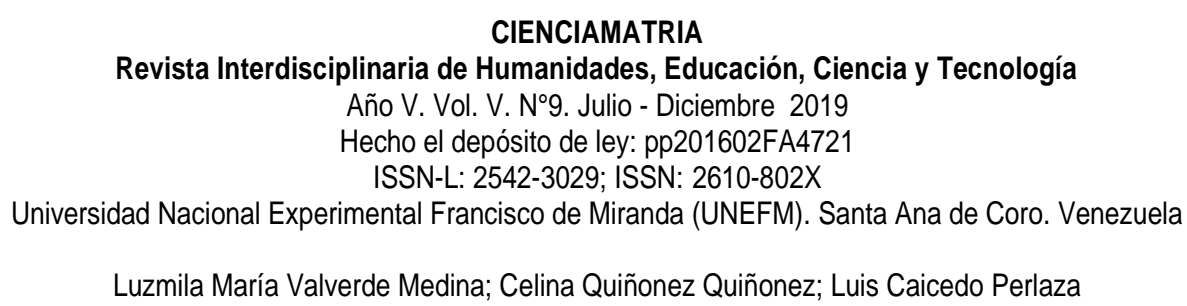

Cabe destacar, que el proceso de visión y de cambio que persigue el líderente, unido al manejo de la misión de administrar los recursos financieros, técnicos y humanos y la responsabilidad de la gerencia, da origen a una forma de practicar el liderazgo y la gerencia, la cual indica la inclinación actual de desempeño, en este caso particular del gerente universitario.

Empatía: es una habilidad fundamental dentro de las diferentes profesiones, especialmente en aquéllas que tienen que ver con el trato al público, las ventas, las Relaciones Públicas, los recursos humanos, por citar algunas. Battoni (2006), afirma que la empatía "es la habilidad para entender las necesidades, sentimientos y problemas de los demás, poniéndose en su lugar, y responder correctamente a sus reacciones emocionales". (p.67). Comprende la capacidad de escuchar a los demás y entender sus problemas y motivaciones. Se refiere a la captación de sentimientos, necesidades e intereses ajenos. Está compuesta por:

Comprender a los demás: saber percibir, reconocer sentimientos, perspectivas en los demás, escuchando abiertamente y estando atento a pistas emocionales.

Ayudar a los demás: percibir las necesidades de desarrollo de otros, reconociendo, recompensando las virtudes, logros y progresos a la vez ofrecer orientaciones que sirvan para fortalecer y alentar las habilidades del otro.

Orientación de servicio: es la habilidad desarrollada para saber detectar las redes cruciales, así como sus corrientes emocionales y su relación de poder. Consiste en reconocer con precisión la realidad externa

Para Bizquera (2009), la empatía es la habilidad que posee un individuo de inferir los pensamientos y sentimientos de otros, lo que genera sentimientos de simpatía, comprensión y ternura, muchas disciplinas las han considerado un fenómeno muy importante. Igualmente, para Goleman (2010), la empatía es el conjunto de capacidades que permiten reconocer y entender las emociones de los demás, sus motivaciones y las razones que explican su comportamiento. En otras palabras, supone poder ponerse en 


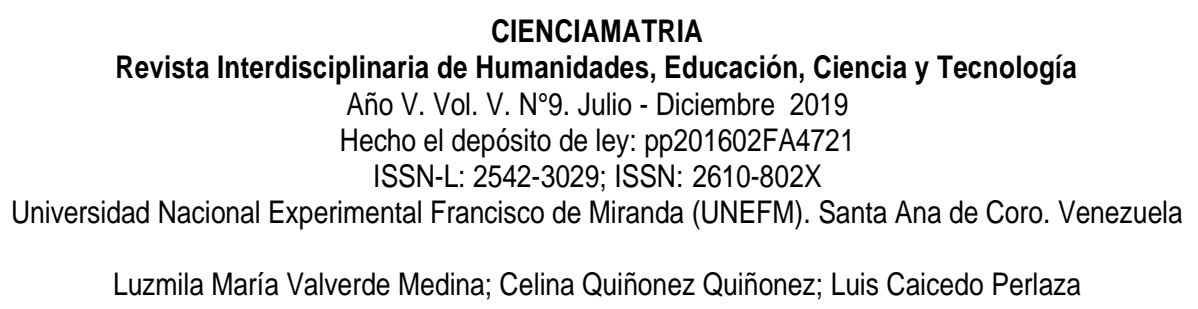

el lugar del otro, pero por supuesto no mimetizándose con él sino desde un lugar discriminado. Es decir, se puede entender al otro y compartir su alegría o su dolor sin necesariamente sentir que a uno le pasa lo mismo.

Con base a lo plasmado se infiere que la empatía es una característica relevante dentro de las Relaciones Púbicas, pues esta constituye la disciplina que tradicionalmente se ha ocupado en forma más directa del estudio y posterior programación del intercambio entre organización y sus públicos de forma planificada. Puesto que su objetivo primordial es establecer, mantener y optimizar las relaciones de la organización con sus públicos por ello es importante siempre ponerse en el lugar de las demás personas para poder ser un buen puente entre ambas, logrando así una comunicación efectiva.

Creatividad: es una cualidad importante dentro de las Relaciones Públicas, pues este profesional debe ser creativo para innovar los planes y estrategias que la organización o institución requieran, debe estar informado de la actualidad constantemente. La creatividad hace referencia específicamente al conjunto de etapas o pasos que suceden desde el inicio antes de la generación de la idea hasta la elaboración final de la misma. Al respecto, el autor García (2003), refiere que el proceso creativo se concibe como el conjunto de etapas y actividades que desarrollan las personas durante el proceso creador.

Seguidamente, Picado (2006), explica que por creatividad se entiende principalmente hacer algo nuevo y diferente, hacer en el sentido de componer, producir, mejorar. Gran parte del proceso creativo es de naturaleza intuitiva e implica un trabajo mental previo a una gestación consciente. El nivel inicial del proceso creativo se describe como un momento de esfuerzo mental, de preparación o percepción de una necesidad. Se trata de un trabajo preparatorio, muy profundo, que hace posible al individuo ir hacia la incubación o gestación de lo que se quiere, hasta hacer evidente posteriormente su logro. Por otra parte, Rodríguez (2014), asegura que la creatividad no es un tema abstracto, la creatividad se presenta en la cabeza de cada uno de los componentes de las 


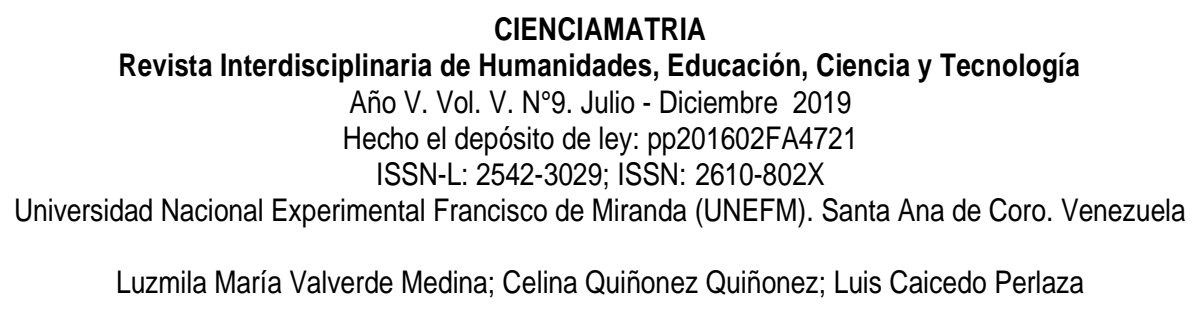

organizaciones. La generación de ideas individuales fecunda, a través de la comunicación y el análisis, a cada uno de los miembros de los equipos humanos para obtener ideas de mayor calidad y trascendencia.

Trabajo en equipo: el éxito en las relaciones públicas se basa en un trabajo en equipo, solo de esta manera las estrategias de comunicación dentro de las instituciones se aprovecharán al máximo y lograrán ser eficientes. Reza (2009), refiere que un equipo de trabajo es un conjunto de personas altamente organizadas y orientadas hacia la consecución de una tarea común. Lo que un número reducido de personas que adoptan e intercambia roles y funciones con flexibilidad de acuerdo con normas preestablecidas, y que disponen de habilidades para manejar sus relaciones con otras personas en un clima de mutuo respeto y confianza.

Dentro de la misma perspectiva, De la Cruz (2014), señala que la capacidad del trabajo en equipo es la habilidad para colaborar e interactuar participando activamente en la consecución de un fin concreto. Así pues, trabajar en equipo requiere una disposición a compartir metas y anteponerlas a los propios intereses personales. Trabajar en equipo requiere hacerlo de forma complementaria, aunando esfuerzos y aportando las competencias propias para la consecución de un objetivo común, siendo conscientes que el todo es más que la suma de las partes.

Responsabilidad: es un estado que deben conservar las personas que realizan una actividad, cualquiera que sea su naturaleza. Es el sentimiento de saber con certeza cuál es su trabajo aunado a la función dentro de las instituciones o empresas, es importante resaltar, que es como una actitud de todos los individuos que tienen ética y valores.

En este contexto, Serna (2005) define la responsabilidad como "el poder que se le asigna a una persona o a un grupo que es consciente de que tiene dominio, el control y el poder sobre su trabajo".(p.78). Es decir, es un factor que debe estar introducido en el ser de cada uno de los trabajadores de las instituciones u organizaciones, de forma que lo guie 
a cumplir con eficiencia y eficacia el desempeño las labores de manera que cree satisfacción por el compromiso realizado.

Igualmente, se menciona que la responsabilidad dentro del puesto de trabajo no debe verse como un derecho sino como un deber. Por ello, Gutiérrez (2007) indica que "la obligación que los miembros de las organizaciones tienen para realizar el trabajo asignado o asegurarse que alguien más lo cumpla en la forma ordenada". (p.104). En este sentido, se concibe por responsabilidad del cumplimiento íntegro del relacionista público en las labores o deberes específicos en que están trabajando.

\section{METODOLOGIA}

El estudio considero una metodologia de tipo descriptiva documental, no experimental, transaccional y de campo. La muestra fue de tipo censal. Para desarrollar la investigación se aplicó un instrumento tipo cuestionario contentivo de 25 preguntas y se utilizó un registro anecdótico, que reúno un relato descriptivo de los gerentes investigados. E instrumento fue sometido a la validez de contenido y al cálculo de la confiabilidad con un valor de 0.85 , considerado altamente confiable. Para el análisis de los datos cuantitativos se aplicó la estadística descriptiva inferencial a través del uso de un paquete estadístico.

\section{RESULTADOS}

Los resultados del análisis de los datos aportados por los investigados, se presentan en la siguiente tabla con sus respectivos análisis:

Análisis frecuencial y porcentual del perfil publico relacionista del gerente educativo universitario Tabla 1.

\begin{tabular}{c|c|c|c|c|c|c|c|c|c|c|}
\hline Alternativas & \multicolumn{2}{|c|}{ Siempre } & \multicolumn{2}{c|}{$\begin{array}{c}\text { Casi } \\
\text { Siempre }\end{array}$} & $\begin{array}{c}\text { Algunas } \\
\text { Veces }\end{array}$ & \multicolumn{2}{c|}{$\begin{array}{c}\text { Casi } \\
\text { Nunca }\end{array}$} & \multicolumn{2}{|c|}{ Nunca } \\
\hline Estadística & Fr & $\%$ & Fr & $\%$ & Fr & $\%$ & Fr & $\%$ & Fr & $\%$ \\
\hline Liderente & 2 & 10 & 4 & 20 & 10 & 50 & 3 & 15 & 1 & 5 \\
\hline
\end{tabular}


CIENCIAMATRIA

Revista Interdisciplinaria de Humanidades, Educación, Ciencia y Tecnología

Año V. Vol. V. No9. Julio - Diciembre 2019

Hecho el depósito de ley: pp201602FA4721

ISSN-L: 2542-3029; ISSN: 2610-802X

Universidad Nacional Experimental Francisco de Miranda (UNEFM). Santa Ana de Coro. Venezuela

Luzmila María Valverde Medina; Celina Quiñonez Quiñonez; Luis Caicedo Perlaza

\begin{tabular}{c|c|c|c|c|c|c|c|c|c|c} 
Empatía & 3 & 15 & 9 & 45 & 6 & 30 & 2 & 10 & 0 & 0 \\
\hline Creatividad & 1 & 5 & 2 & 10 & 11 & 55 & 3 & 15 & 3 & 15 \\
\hline $\begin{array}{c}\text { Trabajo en } \\
\text { Equipo }\end{array}$ & 3 & 15 & 4 & 20 & 12 & 60 & 1 & 5 & 0 & 0 \\
\hline $\begin{array}{c}\text { Responsabilid } \\
\text { ad }\end{array}$ & 8 & 40 & 7 & 35 & 5 & 25 & 0 & 0 & 0 & 0 \\
\hline $\begin{array}{c}\text { Promedio del } \\
\text { Perfil publico } \\
\text { Relacionista } \\
\text { del gerente }\end{array}$ & 3 & 15 & 5 & 25 & 9 & 45 & 2 & 10 & 1 & 5 \\
\hline
\end{tabular}

Fuente: Elaboración propia (2019)

Análisis frecuencial y porcentual del perfil publico relacionista del gerente educativo universitario.

Grafico 1

\section{Título del gráfico}

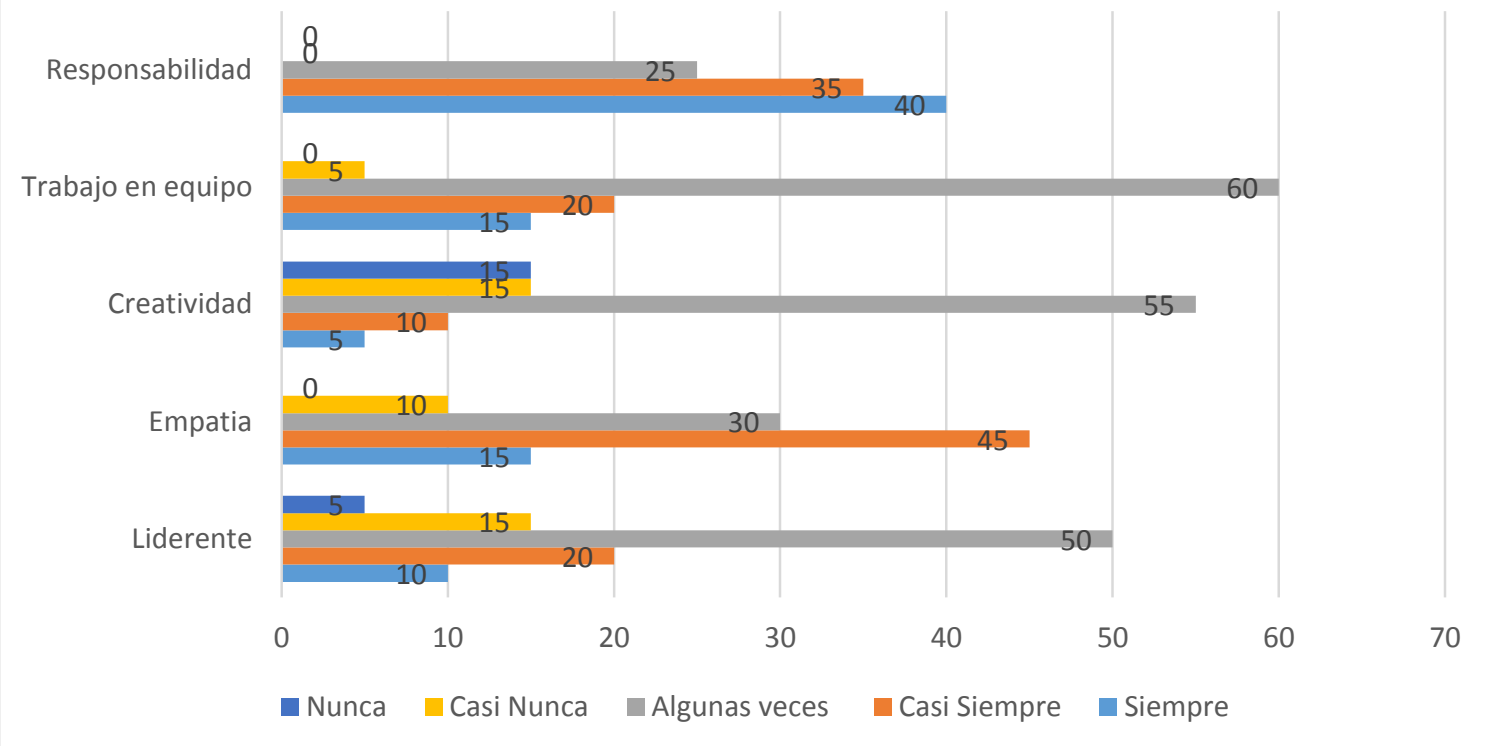

Fuente: Elaboración propia (2019)

En la tabla y grafico 1, se indican los resultados del análisis descriptivo del perfil público relacionista del gerente universitario como producto del análisis conjunto de los indicadores Liderente, empatía, creatividad, trabajo en equipo y responsabilidad. En la 


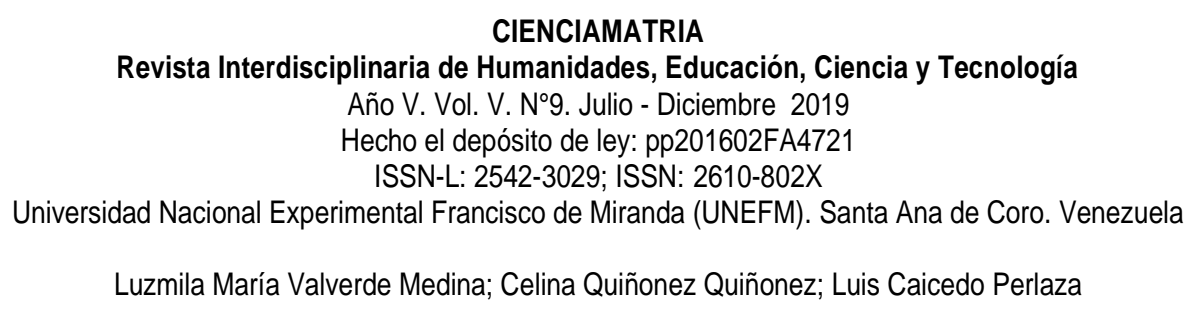

misma se indica que el $45 \%$ de los gerentes universitarios investigados indicaron que algunas veces asumen un perfil público relacionista durante el ejercicio de su cargo.

Con respecto al indicador Liderente, la mayor frecuencia se indicó para algunas veces los gerentes universitarios asumen esta posición con el 50\% de las opiniones, seguida de la opción casi siempre con el $20 \%$ de los casos, todo ello está dado a las habilidades que muestra el gerente como líder, que no siempre ayudan a sus subalternos, para que estos, participen con éxito en la toma estratégica de decisiones.

En cuanto al indicador Empatía, se puede apreciar que la mayor inclinación de repuestas se concentraron en la alternativa casi siempre asumen una posición empática, y con el $30 \%$ de las opiniones para solo algunas veces. Los argumentos para esto fueron que el gerente universitario considera que aún no posee la habilidad para entender las necesidades, sentimientos y problemas del personal con el que le corresponde dirigir y con poca capacidad de escuchar a los demás. Sin embargo, considera que a veces genera entre su entorno laboral sentimientos de simpatía.

Con respecto al indicador Creatividad, el 55\% de los gerentes investigados consideraron que algunas veces ponen en práctica una posición creativa y contrariamente el 35\% considero que nunca lo hace. Este resultado presume evidenciar, que el surgimiento de nuevas ideas no son de mayor trascendencia entre el personal a su cargo.

Ahora bien, en lo que corresponde al indicador Trabajo en Equipo, la alternativa con mayor tendencia fue algunas veces, con el $32,2 \%$ de las respuestas y un $20 \%$ que indico la alternativa casi siempre. Esta posición de los gerentes, permite deducir que el perfil público relacionista está presente para darle cumplimiento solo al seguimiento de alguna determinada tarea laboral y/o administrativa.

En relación al indicador Responsabilidad, se señala que el $40 \%$ de los gerentes universitarios investigados consideraron que siempre asumen una posición responsable ante sus obligaciones y el 35\% indica que casi siempre. Cabe destacar, que estas repuestas no se corresponden al estado que deben conservar los gerentes que realizan 


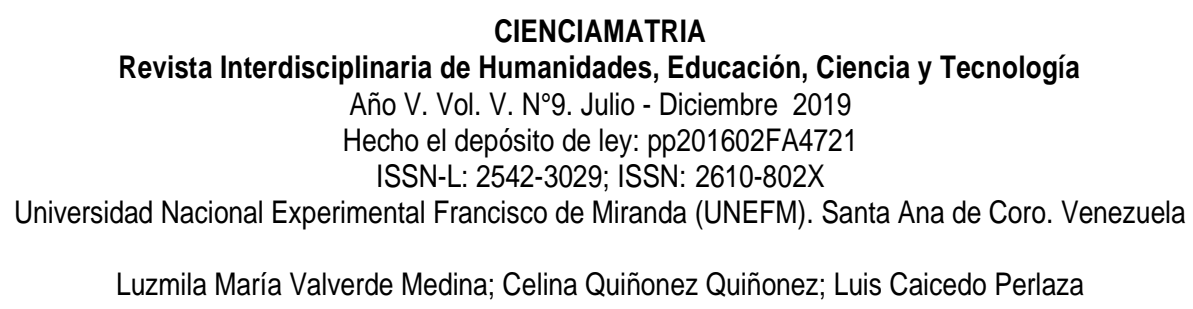

actividades de alta responsabilidad, para asegurar el cumplimiento al trabajo asignado y en forma ordenada.

\section{CONCLUSIONES}

De acuerdo a lo desarrollado en este trabajo, relacionado a con la caracterización del perfil público relacionista del gerente educativo universitario, se presentan las siguientes conclusiones:

Los gerentes universitarios investigados indicaron que algunas veces asumen un perfil público relacionista durante el ejercicio de su cargo, lo que permite evaluarlos con un perfil medianamente aceptable, implicando que se hace imperante una revisión constante de las aptitudes de lederente, empático, creativo, del trabajo en equipo y de sus rasgos de responsabilidad como gerente, para que se ajuste a las necesidades de las organizaciones educativas.

Solo algunas veces se cumplen los indicadores de liderente, creatividad y trabajo en equipo, mientras que casi siempre asumen un perfil público relacionista empático como gerente universitario y con mayor frecuencia de aceptación entre los investigados fue la alternativa siempre irrumpen una posición responsable durante la ejecución de sus funciones como gerente.

\section{REFERENCIAS CONSULTADAS}

1. Alles, M. (2013). Comportamiento Organizacional: Cómo lograr un cambio cultural a través de Gestión por competencias. México: Ediciones Granica

2. Barquero, J. (2012). Manual de Relaciones Públicas Empresariales. España: Ediciones Gestión 2000.

3. Batoni, A (2006). Relaciones Públicas. Barcelona: Ediciones Gestión 2000.

4. Bizquerra, R. (2009). Métodos de Investigación Educativa. México: Editorial CEAC. 


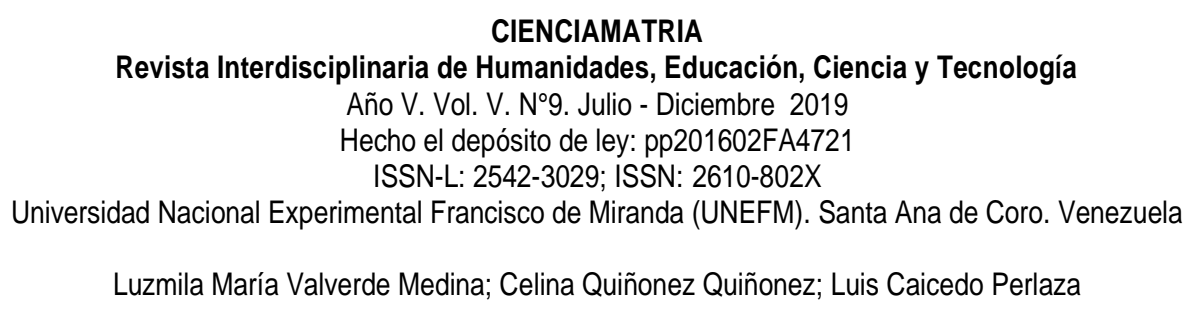

5. De la Cruz, L. (2014). Comunicación efectiva y trabajo en equipo. Ministerio de Educación, Cultura y Deporte

6. García, J. (2003), Relaciones Públicas. Teoría e historia, Universitaria. Barcelona: Universitata Oberta.

7. Gómez, M., \& Salas de González, M. (2018). Inteligencia Emocional del Gerente Educativo y Clima Laboral en Escuelas Bolivarianas Venezolanas. Revista Arbitrada Interdisciplinaria Koinonía, 3(5), 10-26. Recuperado de http://fundacionkoinonia.com.ve/ojs/index.php/revistakoinonia/article/view/111/93

8. Melendez, O (2000). El Liderente Publicitario. Imprenta del Estado de Zulia Maracaibo Venezuela. 1era Edición

9. Meng, J; y Berger, B. (2010). Cultural influence on the perceptions of effective leadership in public relations" comunicación presentada a la 60 Annual Convention of the International Communication Association (ICA)

10. Miguez, M. (2010). Los públicos en las Relaciones Públicas. Barcelona-España: Editorial UOC.

11. Moraima Romero, D., \& Aldana Zavala, J. (2019). Proyectos Comunitarios en el proceso de Transformación Social de la Universidad. CIENCIAMATRIA, 5(9), 151 - 167. https://doi.org/10.35381/cm.v5i9.104

12. Otero, M. (2009). Protocolo y organización de eventos. Barcelona-España: Editorial UOC.

13. Picado, F. (2006). Didáctica Gerencial. Una Perspectiva Integradora. San José de Costa Rica: Euned.

14. Reza, J. (2009). Equipos de trabajo efectivo y altamente productivo. Caracas: Ed. Panorama

15. Rodríguez, J. (2014). Las Relaciones Públicas como herramienta para el Desarrollo de la comunicación externa en asociaciones Civiles. Tesis de Grado. URBE. Maracaibo. 
16. Serna, H. (2005), Gerencia Estratégica. Planeación y Gestión. Teoría y Metodología. Bogotá-Colombia: 3R Editores LTDA.

17. Vargas, L. (2009). Colegio de Periodistas de Costa Rica. Perfil de un relacionista público. [En línea] Fecha de acceso: 27 de agosto del 2016. Disponible en: http://www.colper.or.cr/paginas/bolsaempleo/p_rpubllica.htm

\section{REFERENCES CONSULTED}

1. Alles, M. (2013). Organizational Behavior: How to achieve cultural change through Competency Management. Mexico: Granica Editions

2. Barquero, J. (2012). Business Public Relations Manual. Spain: 2000 Management Editions.

3. Batoni, A (2006). Public relations. Barcelona: 2000 Management Editions.

4. Bizquerra, R. (2009). Educational Research Methods. Mexico: CEAC Editorial.

5. De la Cruz, L. (2014). Effective communication and teamwork. Ministry of Education, Culture and Sports

6. García, J. (2003), Public Relations. Theory and history, University. Barcelona: Universitata Oberta.

7. Gómez, M., \& Salas de González, M. (2018). Emotional Intelligence of the Educational Manager and Labor Climate in Venezuelan Bolivarian Schools. Interdisciplinary Arbitrated Review Koinonía, 3 (5), 10-26. Recovered from http:/fundacionkoinonia.com.ve/ojs/index.php/revistakoinonia/article/view/111/93

8. Melendez, $O$ (2000). The Advertising Leader. Printing of the State of Zulia Maracaibo Venezuela. 1st Edition

9. Meng, J; and Berger, B. (2010). Cultural influence on the perceptions of effective leadership in public relations "communication presented to the 60th Annual Convention of the International Communication Association (ICA) 
10. Miguez, M. (2010). Publics in Public Relations. Barcelona-Spain: Editorial UOC.

11. Moraima Romero, D., \& Aldana Zavala, J. (2019). Community Projects in the process of Social Transformation of the University. SCIENCE, 5 (9), 151-167. Https://doi.org/10.35381/cm.v5i9.104

12. Otero, M. (2009). Protocol and organization of events. Barcelona-Spain: Editorial UOC.

13. Picado, F. (2006). Management Teaching. An Integrative Perspective. San José de Costa Rica: Euned.

14. Reza, J. (2009). Effective and highly productive work teams. Caracas: Ed. Panorama

15. Rodríguez, J. (2014). Public Relations as a tool for the development of external communication in civil associations. Thesis. CITY. Maracaibo

16. Serna, H. (2005), Strategic Management. Planning and Management. Theory and Methodology Bogotá-Colombia: 3R Editores LTDA.

17. Vargas, L. (2009). Association of Journalists of Costa Rica. Profile of a public relationist. [Online] Access date: August 27, 2016. Available at: http://www.colper.or.cr/paginas/bolsaempleo/p_rpubllica.htm 